\title{
Modificações em Codificador de Voz 3G para Compressão de Sinais de Eletromiografia
}

\author{
José Haroldo de Assis Cavalcante e Lúcio Martins da Silva
}

\begin{abstract}
Resumo- O codificador de voz AMR-ACELP foi padronizado para o uso na telefonia celular de terceira geração. Diversas modificações foram aplicadas neste codificador de forma a torná-lo mais eficiente para a compressão de sinais de eletromiografia, produzidos pela contração de músculos voluntários. Conseguiram-se taxas de compressão variando entre $81,4 \%$ a $90,3 \%$ e um erro quadrático médio de $1,8 \%$. Em termos de eficiência, estes resultados ultrapassaram os obtidos por estudo anterior usando o mesmo codificador, e aproximaram-se de outros usando respectivamente wavelets ou H.264/AVC, indicando que a técnica pode ser adaptada com sucesso para uso com os atuais canais de comunicação e hardware disponível para processamento e transmissão de voz.
\end{abstract}

Palavras-chave - Compressão, eletromiografia, AMR-ACELP.

Abstract - The voice encoder AMR-ACELP was standardized for use in the third generation cellular telephony. Several changes were implemented in this codec to make it more efficient for the compression of signals of electromyography, produced by the contraction of voluntary muscles. Compression rates ranging from $81.4 \%$ to $90.3 \%$ and a mean square error of $1.8 \%$ were obtained. In terms of efficiency, these results outperformed those achieved by a previous study using the same encoder, and were close to others using, respectively, wavelets or H.264/AVC, indicating that the technique can be adapted successfully for use with existing communication channels and hardware available for processing and transmission of speech.

Keywords - Compression, electromyography, AMR-ACELP.

\section{INTRODUÇÃO}

A eletromiografia (EMG) consiste na captação e gravação das mudanças no potencial elétrico das fibras musculares durante a sua contração, geralmente com a ajuda de eletrodos. No caso da eletromiografia de superfície (SEMG), os eletrodos são colocados sobre a pele e, geralmente, registra a superposição dos potenciais de um grande número de fibras, produzindo sinais não-periódicos [1], semelhantes a ruído ou a sons surdos da fala. Um sinal SEMG típico possui componentes de frequência até cerca de $1 \mathrm{kHz}$ e amplitude de vários $\mathrm{mV}$, dependendo das características dos eletrodos e dispositivos de captura empregados. Um registro EMG pode durar de alguns segundos a várias horas, conforme a aplicação.

A compressão do sinal EMG é importante para permitir ou facilitar o seu armazenamento, especialmente quando se trata de sinais de longa duração ou de grandes bases de dados, ou a

José Haroldo de Assis Cavalcante e Lúcio Martins da Silva, Departamento de Engenharia Elétrica, Universidade de Brasília, Brasília, DF, Brasil. E-mails: joseharoldo@unb.br, lucio@ene.unb.br. sua transmissão por meio de redes de banda limitada. Várias técnicas de compressão EMG já foram propostas, baseadas em transformada e predição linear. Este artigo apresenta uma investigação sobre o uso do codificador de voz AMR-ACELP [2]-[5] para a compressão de sinais SEMG. Foram criados novos modos de operação para o codec AMR original, que foram testados em um grupo de sinais SEMG e em um sinal de voz de referência, efetuando-se sequencialmente a compressão (codificação) e a reconstrução (decodificação) de cada sinal.

\section{O CODEC AMR-ACELP}

O codec AMR-ACELP foi padronizado pelo 3GPP e pelo ETSI como o padrão de codificação de voz dos sistemas de terceira geração de telefonia celular baseados na evolução do sistema GSM [2]. Esse codec utiliza o esquema ACELP (Algebraic Code Excited Linear Prediction) [3]. A sua estrutura básica é descrita sucintamente a seguir - descrições detalhadas podem ser encontradas em [2]-[5]. A Figura 1 mostra o diagrama de blocos simplificado do codificador CELP, que é a base do codec AMR. O modelo de síntese do codec AMR-ACELP é constituído de um filtro linear excitado por dois dicionários: um dicionário fixo do tipo algébrico [3] e um adaptativo.

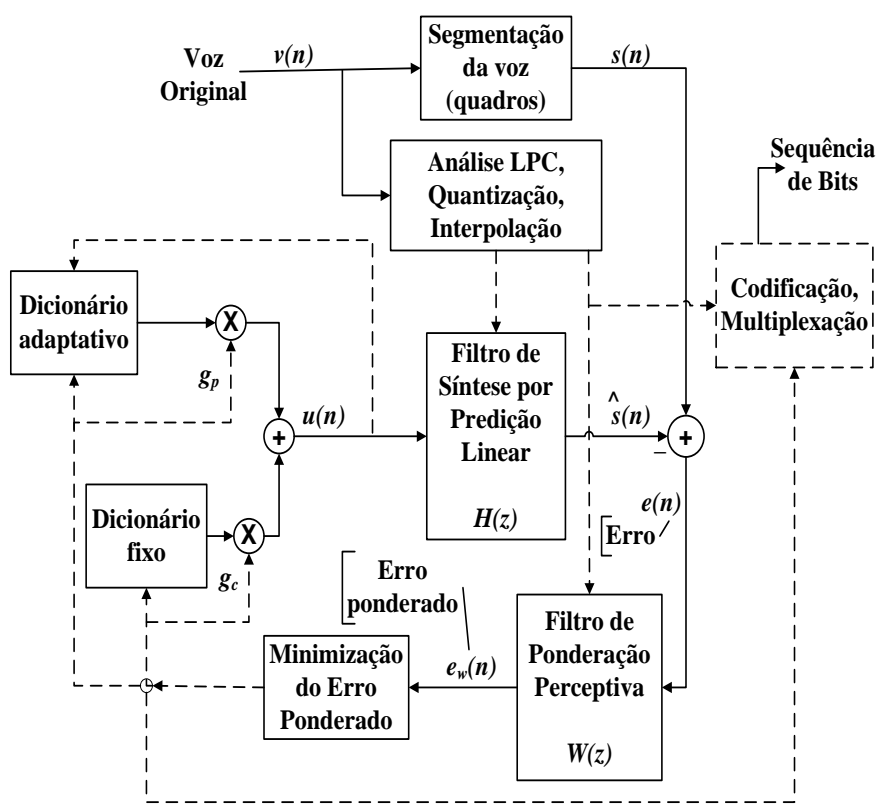

Fig. 1 -.Diagrama de blocos simplificado de um codificador CELP.

O dicionário fixo do codec AMR-ACELP é constituído de vetores formados apenas por pulsos com valores 0 (em sua maioria), -1 ou +1 , o que reduz o esforço computacional 
relacionado à busca do vetor [3]. O dicionário adaptativo consiste de versões deslocadas no tempo das sequências de excitação passadas e modela a correlação de longo prazo do sinal de voz - ele substitui um filtro de síntese de longo termo. $\mathrm{O}$ filtro de síntese por predição linear de curto termo modela a envoltória espectral de cada segmento de voz codificado.

A sequência de excitação ótima em um dicionário é escolhida usando um procedimento de busca de análise-porsíntese, em que é minimizado o valor quadrático médio do erro $e_{w}(n)$, uma versão ponderada perceptivamente do erro $e(n)$, entre a voz original e a sintetizada. Para reduzir o esforço computacional, os dicionários adaptativo e fixo são varridos sequencialmente em busca das melhores sequências de excitação.

O filtro de ponderação do erro, $W(z)$, é utilizado para melhorar a qualidade subjetiva da voz reconstruída posteriormente pelo decodificador.

O AMR opera sobre quadros de voz de $20 \mathrm{~ms}$, correspondendo a 160 amostras na taxa de amostragem de 8.000 amostras/s. A cada 160 amostras, o sinal de voz é analisado para extração dos parâmetros do modelo CELP, que são codificados e transmitidos. No decodificador, os parâmetros são decodificados e a voz é reconstruída por meio de um filtro de síntese preditivo linear.

Codecs CELP tradicionais utilizam uma taxa fixa de codificação. Em contraste, o AMR, como resultado da alocação de quantidades diferentes de bits para cada conjunto de parâmetros, é um codec multitaxa integrado, com oito modos de operação, com taxas de codificação variando de $4,75 \mathrm{kbit} / \mathrm{s}$ (modo AMR_4.75) a 12,2 kbit/s (modo AMR_12.20) [2]. Por exemplo, os modos AMR_12.20, AMR_10.20 e AMR_7.95 utilizam, respectivamente, 244, 204 e 159 bits/quadro. A denominação de cada modo indica a taxa de bits despendida na codificação.

\section{VERSÃO DO AMR-ACELP ADAPTADA PARA SINAIS SEMG}

Várias mudanças foram feitas no codec AMR original descrito em [4] e [5], visando a melhoria do seu desempenho com o sinal EMG [9]. Alguns procedimentos que propiciam a melhoria subjetiva da voz reconstruída foram retirados e se testou novas combinações de dicionários, incluindo a retirada do dicionário adaptativo. Essas mudanças levaram a oito novos modos de operação. Estas mudanças e as características dos novos modos são detalhadas a seguir.

Entre os oito modos originais do AMR, o AMR_12.20 e o AMR_7.95 foram selecionados como fontes para a criação de novos modos. O modo AMR_12.20 oferece a mais alta qualidade para o sinal de voz reconstruído, seguido pelo modo AMR_10.20 e depois pelo modo AMR_7.95. No entanto, as estruturas dos modos AMR_12.20 e AMR_7.95 são mais semelhantes entre si do que com a estrutura do modo AMR_10.20. Especialmente, os modos AMR_12.20 e AMR_7.95 são os únicos modos originais que codificam separadamente os ganhos dos dicionários fixo e adaptativo. Isso facilita mudanças e combinações de partes desses módulos.

\section{A. Retirada dos Procedimentos para Melhoria Subjetiva da Voz}

Vários procedimentos do codec AMR-ACELP propiciam a melhoria da qualidade subjetiva do sinal de voz reconstruído, embora reduzam a similaridade entre a forma de onda desse sinal e a forma de onda do sinal de voz original. Contudo, essa melhoria subjetiva não tem qualquer significado para os sinais de EMG e, em princípio, o que importa é a similaridade entre as formas de onda dos sinais reconstruído e original. Assim, tais procedimentos foram retirados nos novos modos do codec.

Um dos procedimentos retirados foi a ponderação do erro pelo filtro $W(z)$. Isto é, a otimização no processo de análise por síntese passou a minimizar diretamente o valor quadrático médio do erro $e(n)$. Ainda no codificador, foi retirada a préfiltragem adaptativa aplicada no vetor-código selecionado no dicionário fixo - essa pré-filtragem realça os componentes espectrais nas vizinhanças das frequências múltiplas do inverso do atraso tonal (pitch) determinado para o dicionário adaptativo.

No decodificador, foi retirada a pós-filtragem adaptativa, que reforça os componentes de frequiência nas proximidades dos formantes de voz. Retirou-se também a suavização do ganho do dicionário fixo, cuja função é evitar flutuações súbitas na intensidade do sinal entre quadros, o processamento anti-esparsidade que é aplicado ao vetor-código do dicionário fixo e o pós-processamento aplicado à excitação para enfatizar a contribuição do dicionário adaptativo na voz sintetizada.

Foram retirados ainda os filtros passa-altas utilizados na entrada do codificador e na saída do decodificador, porque as frequências de corte desses filtros não são adequadas para sinais EMG.

\section{B. Retirada do Dicionário Adaptativo}

O dicionário adaptativo é particularmente eficiente para modelar os sons sonoros da fala, que são sinais quaseperiódicos. Por outro lado, a contribuição desse dicionário não é relevante na representação dos sons surdos da fala. As formas de onda dos sinais de SEMG são muito parecidas com as formas de onda dos sons surdos da fala. Assim, já era esperado que o dicionário adaptativo não seria eficiente para representar os sinais de SEMG. Isso foi confirmado pelos resultados das simulações, que serão apresentados posteriormente.

\section{Utilização de Dois Dicionários Fixos}

$\mathrm{Na}$ maioria dos novos modos simulados, o dicionário adaptativo foi removido, pelo motivo apresentado anteriormente. Em alguns desses modos, o codec ficou então com um único dicionário. Contudo, em outros modos, um segundo dicionário fixo foi acrescentado. Em todos os novos modos que possuem dois dicionários, o procedimento de busca utilizado foi o sequencial.

\section{Resumo dos Novos Modos}

Como já foi dito, os novos modos testados com os sinais de SMEG foram construídos a partir dos modos originais AMR_12.20 e AMR_7.95 [2], [3]. A Tabela I contém uma lista dos novos modos, com a indicação do modo de origem e o dispêndio de bits com cada quadro de 160 amostras do sinal SEMG. A designação dos novos modos é feita com dois ou três campos. O primeiro indica quantos conjuntos de coeficientes LP (linear prediction) são transmitidos para cada 
quadro. O segundo campo especifica o primeiro dicionário do codec, que pode ser o dicionário adaptativo (DA) ou um dicionário fixo (DF) — nesse caso, é especificada a quantidade de pulsos não-nulos em cada vetor-código de dimensão 40 (comprimento de um sub-quadro). O terceiro campo especifica o segundo dicionário, se ele existir. $\mathrm{Na}$ composição dos novos modos, foram utilizados dois dicionários algébricos: com 10 (DF10) e 4 (DF4) pulsos nãonulos. Esses são os dicionários dos modos originais AMR_12.20 e AMR_7.95, respectivamente. A Figura 2 esquematiza as relações entre os modos originais do AMR e os novos modos construídos para sinais de SEMG.

TABELA I

NOVOS MODOS DE CODIFICAÇÃO PARA SINAIS SEMG.

\begin{tabular}{|l|c|c|}
\hline \multicolumn{1}{|c|}{ Modo } & Origem & Bits/quadro \\
\hline 2LP/DA/DF10 & AMR_12.20 & 244 \\
\hline 2LP/DF10 & AMR_12.20 & 198 \\
\hline 2LP/DF10/DF10 & AMR_12.20 & 358 \\
\hline 1LP/DA/DF10 & AMR_7.95 & 231 \\
\hline 1LP/DF10 & AMR_7.95 & 187 \\
\hline 1LP/DF10/DF4 & AMR_7.95 & 275 \\
\hline 1LP/DF10/DF10 & AMR_7.95 & 347 \\
\hline 1LP/DF4/DF4 & AMR_7.95 & 203 \\
\hline
\end{tabular}

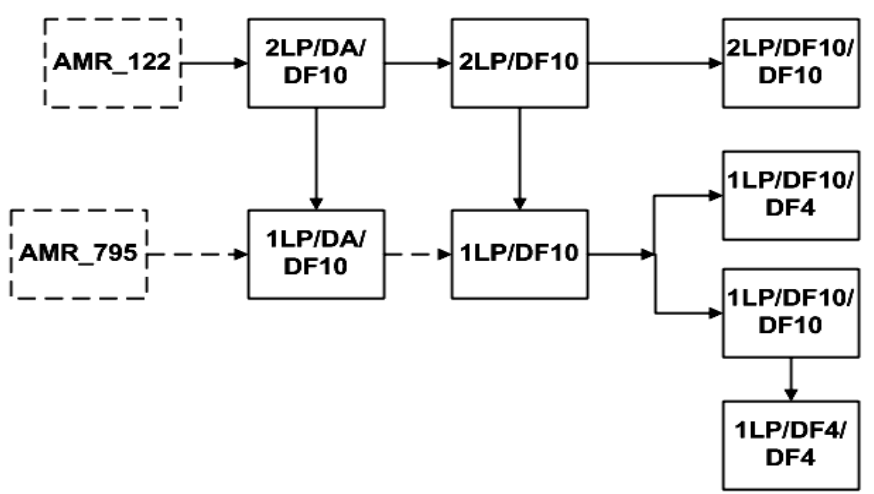

Fig. 2 - Relação entre modos originais e novos modos.

O novo modo 2LP/DA/DF10 difere do modo original AMR_12.20 apenas pela eliminação dos procedimentos para melhorar a qualidade subjetiva do sinal de voz reconstruído (veja subseção III- $A$ ). Foram mantidos o esquema de codificação dos coeficientes LP (com a transmissão de dois conjuntos por quadro), os dicionários adaptativo e fixo e, consequentemente, a alocação de bits para um quadro de 160 amostras. $\mathrm{O}$ modo $2 \mathrm{LP} / \mathrm{DF} 10$ foi obtido do modo 2LP/DA/DF10 retirando-se o dicionário adaptativo. O modo 2LP/DF10/DF10 foi obtido do modo 2LP/DF10 acrescentando-se um segundo dicionário fixo idêntico ao primeiro.

O modo 1LP/DA/DF10 foi construído a partir do modo original AMR_7.95, que codifica e transmite um único conjunto de coeficientes LP por quadro. Contudo, o modo AMR_7.95 utiliza o dicionário fixo DF4, que foi substituído pelo DF10. O modo 1LP/DF10 foi obtido do modo 1LP/DA/DF10 retirando-se o dicionário adaptativo. Os modos 1LP/DF10/DF4 e 1LP/DF10/DF10 foram obtidos do modo 1LP/DF10 acrescentando-se um segundo dicionário fixo DF4 ou DF10, respectivamente. O modo 1LP/DF4/DF4 foi obtido do modo 1LP/DF10/DF4 trocando o dicionário DF10 pelo DF4.

\section{SIMULAÇÕES}

\section{A. Sinais Utilizados nos Testes}

Nos testes, utilizou-se doze sinais eletromiográficos, produzidos durante a contração isométrica do músculo biceps brachii, a $60 \%$ da contração máxima voluntária (CMV) - são os mesmos sinais utilizados no estudo descrito em [6]. Esses sinais foram coletados com eletrodos de superfície préamplificados, tendo sido amostrados a uma taxa de 2000 amostras/s e quantizados com resolução de 12 bits — portanto, a taxa de bits é de $24 \mathrm{kbit} / \mathrm{s}$. A eliminação de trechos sem atividade muscular no início e no final de cada captura resultou em sinais com duração entre 61 e 110 segundos. Os sinais foram passados por um filtro passa-faixa digital FIR de fase linear, com frequências de corte inferior e superior de 20 $\mathrm{Hz}$ e $500 \mathrm{~Hz}$, respectivamente.

Para fins de comparação, se utilizou também um sinal de voz com duração de 3 segundos, que foi amostrado à taxa de $8000 \mathrm{~Hz}$ e quantizado com resolução de 16 bits.

Em todos os casos, os sinais foram previamente normalizados pelo valor máximo absoluto, de modo a ocuparem a faixa de valores [-32767 +32767] e gravados em formato PCM de 16 bits, compatível com o formato de arquivo de entrada do codec.

\section{B. Avaliação do Desempenho}

O desempenho dos codecs foi mensurado objetivamente por meio de três critérios usualmente empregados na avaliação da compressão de sinais eletrofisiológicos [6,7]: fator de compressão (compression factor, CF), diferença percentual residual (percent residual difference, PRD) e erro quadrático médio normalizado (mean square error, MSE). O fator de compressão é definido por:

$$
C F=\frac{N_{\text {orig }}-N_{\text {comp }}}{N_{\text {orig }}} \times 100 \%,
$$

onde $N_{\text {orig }}$ e $N_{\text {comp }}$ são as quantidades de bits despendidas para representar o sinal de SEMG sem e com compressão, respectivamente. A Tabela II mostra a taxa de bits e o fator de compressão dos modos de codificação para sinais de SEMG.

TABELA II

TAXA DE BITS E FATOR DE COMPRESSÃO DOS MODOS DE CODIFICAÇÃO PARA SINAIS SEMG.

\begin{tabular}{|l|c|c|}
\hline \multicolumn{1}{|c|}{ Modo } & $\begin{array}{c}\text { Taxa de bits } \\
\text { (kbit/s) }\end{array}$ & $\begin{array}{c}\text { Fator de Compressão } \\
(\mathbf{\%})\end{array}$ \\
\hline 2LP/DA/DF10 & 3,05 & 87,29 \\
\hline 2LP/DF10 & 2,475 & 89,69 \\
\hline 2LP/DF10/DF10 & 4,475 & 81,35 \\
\hline 1LP/DA/DF10 & 2,8875 & 87,97 \\
\hline 1LP/DF10 & 2,3375 & 90,26 \\
\hline 1LP/DF10/DF4 & 3,4375 & 85,68 \\
\hline 1LP/DF10/DF10 & 4,3375 & 81,93 \\
\hline 1LP/DF4/DF4 & 2,5375 & 89,43 \\
\hline
\end{tabular}


A diferença residual percentual (PRD) e o erro quadrático médio percentual (MSE) são definidos, respectivamente, como:

$$
\begin{gathered}
P R D=\sqrt{\frac{\sum_{n=0}^{N-1}[x(n)-\hat{x}(n)]^{2}}{\sum_{n=0}^{N-1} x^{2}(n)}} \times 100 \% \\
M S E=\frac{\sum_{n=0}^{N-1}[x(n)-\hat{x}(n)]^{2}}{\sum_{n=0}^{N-1} x^{2}(n)} \times 100 \%
\end{gathered}
$$

onde $x(n)$ é o sinal original, $\hat{x}(n)$ o sinal reconstruído e $N$ o número de amostras do sinal. Note que a PRD pode ser obtida do MSE e vice-versa.

\section{RESULTADOS DAS SIMULAÇÕES}

\section{A. Desempenho do AMR-ACELP Original}

Para comparação, se processou os sinais de SEMG e o sinal de voz com o modo original AMR_12.20, o melhor dentre os modos originais. As Figuras $3 a$ e $3 b$ mostram as formas de ondas de um segmento de um sinal de SMEG e da sua versão reconstruída pelo AMR12.20, respectivamente. As Figuras 4a e $4 \mathrm{~b}$ mostram formas de onda de um segmento do sinal voz. Note a baixa similaridade entre as formas de onda do sinal original e do sinal reconstruído. A PRD para o sinal de voz foi de $78,3 \%$ e para os sinais de SEMG de $96,7 \%$ (valor médio). Esses valores tão altos são devidos aos procedimentos utilizados para melhorar a qualidade subjetiva do sinal de voz reconstruído.

\section{B. Desempenho do AMR-ACELP Modificado}

Os sinais de SEMG e de voz foram processados utilizando cada um dos novos modos de codificação descritos anteriormente. Como ilustração, as Figuras 3c e 4c mostram as formas de onda do sinal reconstruído pelo modo 2LP/DF10/DF10. Note que agora as formas de onda originais (Figuras $3 \mathrm{a}$ e $4 \mathrm{a}$ ) foram reproduzidas de forma muito mais acurada.

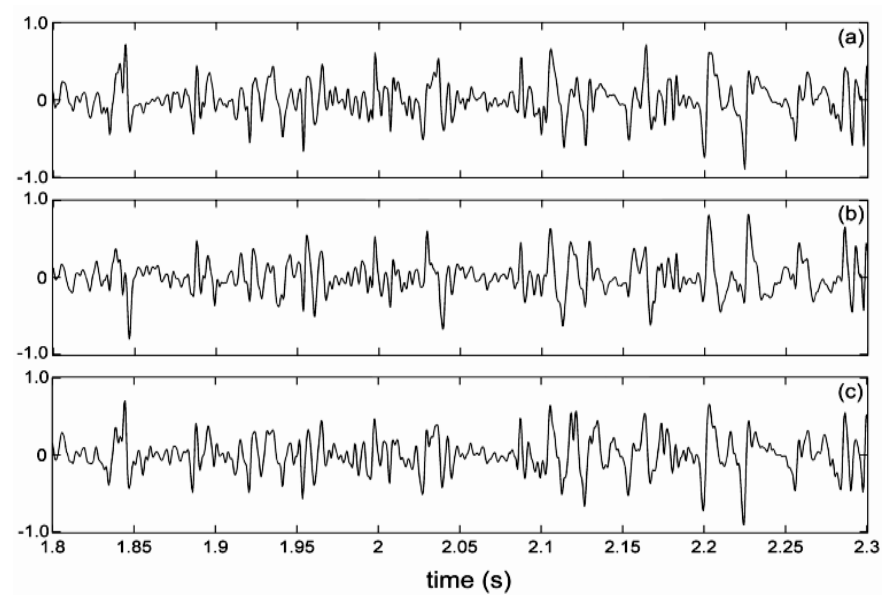

Fig. 3 - Segmentos de sinal SEMG: (a) original; (b) reconstruído pelo modo AMR_12.20, (c) reconstruído pelo modo 2LP/DF10/DF10.

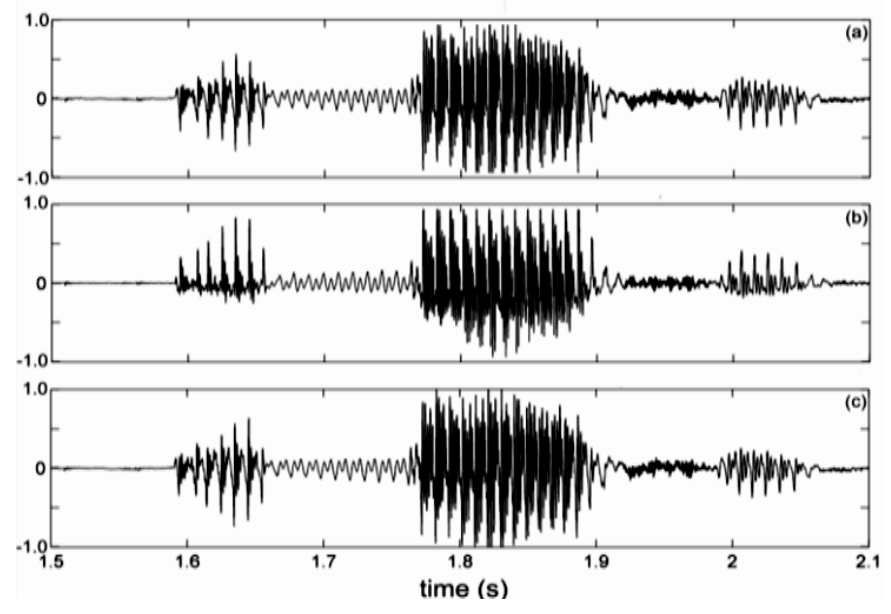

Fig. 4 - Segmentos de sinal de voz: (a) original; (b) reconstruído pelo modo AMR_12.20, (c) reconstruído pelo modo 2LP/DF10/DF10.

A Figura 5 mostra os valores médios de PRD obtidos com os novos modos de codificação para os sinas de SEMG e para o sinal de voz. A Figura 6 mostra a PRD versus a CF, obtida com cada um novos modos para cada sinal de SEMG de teste.

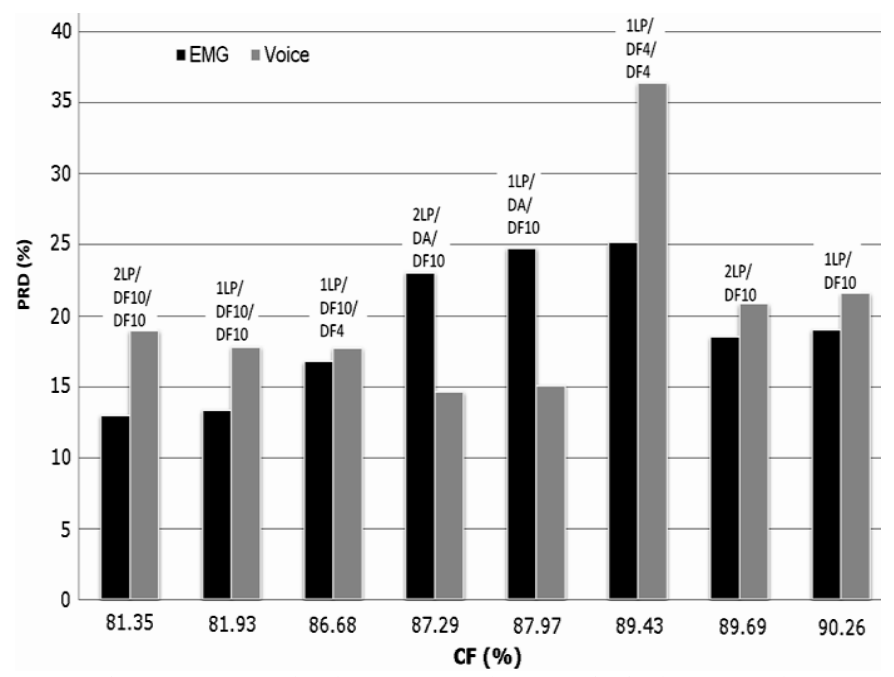

Fig. 5- Desempenho dos novos modos para sinais de EMG e de voz.

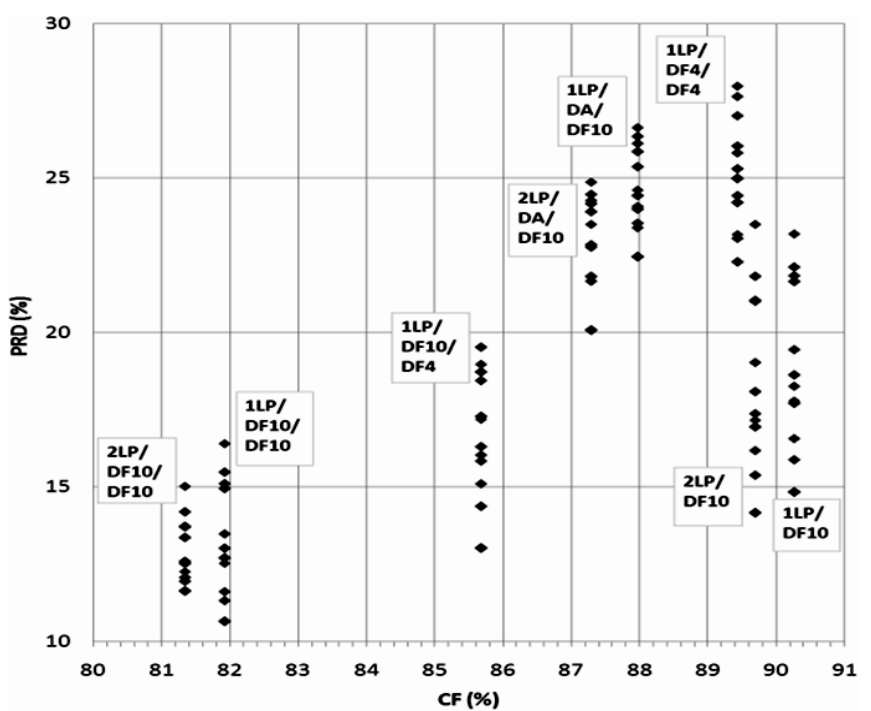

Fig. 6 - Relação entre erro (PRD) e fator de compressão (CF) para os novos modos com sinais de EMG. 


\section{Efeito da Eliminação dos Procedimentos para Melhorar a Qualidade Subjetiva da Voz}

Para o sinal de voz, a PRD obtida com o modo AMR_12.20 foi de 78,3\% e com o modo 2LP/DA/DF10, de 14,6\%. Para os sinais de SEMG, a PRD média foi de $96,7 \%$ e de $23,0 \%$, respectivamente. Esses valores mostram, novamente, o efeito dos procedimentos para melhorar a qualidade subjetiva de voz sobre a qualidade objetiva da forma de onda do sinal reconstruído .

\section{Efeito da Retirada do Dicionário Adaptativo}

A comparação dos desempenhos dos modos 2LP/DA/DF10 e 1LP/DA/DF10 com os desempenhos dos seus modos derivados 2LP/DF10 e 1LP/DF10 — veja Figura 5 - mostra a eficácia do dicionário adaptativo para sinais de voz e sua ineficácia para sinais de SMEG. Para o sinal de voz, a retirada do dicionário adaptativo causou um aumento significativo na PRD, embora tenha aumentado também o fator de compressão (CF). Contudo, para os sinais de SEMG, houve uma redução da PRD: isto é, além de aumentar o CF (devido à retirada de bits de codificação dos parâmetros do dicionário adaptativo), melhorou a qualidade do sinal reconstruído. Isso parece inconsistente: em princípio, seria esperado que a PRD também aumentasse para os sinais de SEMG. A explicação para esse fenômeno está provavelmente no procedimento de busca sequiencial, que é sub-ótimo. Quando se utiliza os dois dicionários, primeiro se determina os parâmetros do dicionário adaptativo, considerando nula a contribuição do dicionário fixo. Em seguida, se determina o melhor vetor-código do dicionário fixo [10]. Se os dois dicionários fossem pesquisados simultaneamente, o referido fenômeno certamente não ocorreria.

\section{E. Efeito da Transmissão de Dois Conjuntos de Parâmetros LP}

Comparando os desempenhos dos modos 1LP/DF10 e 2LP/DF10 e dos modos 1LP/DF10/DF10 e 2LP/DF10/DF10, se vê que a transmissão de dois conjuntos de parâmetros LP por quadro, em vez de um conjunto, propicia uma pequena redução na PRD, mas causa, em compensação, também uma pequena redução no $\mathrm{CF}$ - 11 bits são despendidos para representar o segundo conjunto [4]. Assim, pode ser que o aumento da complexidade computacional não justifique a transmissão de dois conjuntos de parâmetros LP por quadro.

\section{F. Uso de Dois Dicionários Fixos}

Para melhorar a qualidade do sinal reconstruído pode-se aumentar o tamanho do dicionário fixo ou adicionar um segundo dicionário fixo. Nos testes realizados, se limitou a combinações com os dois dicionários algébricos DF4 e DF10, que são os dicionários dos modos originais AMR_12.20 e AMR_7.95, respectivamente.

\section{G. Comparação com Outro Ensaio Usando o AMR-ACELP}

Carotti et al [7] também experimentaram o codec AMRACELP na codificação de sinais de SEMG. Contudo, eles se limitaram a experimentar uma versão pouco modificada do modo AMR_12.20. Eles retiraram a filtragem passa-altas na entrada do codificador e a pós-filtragem do decodificador. No artigo [7], nenhum comentário é feito sobre o filtro de ponderação $W(z)$, embora seja provável que esse tenha sido retirado também. Contudo, o dicionário adaptativo foi mantido.

Foram utilizados sinais de EMG de superfície, produzidos por contrações isométricas variando entre 10 e $70 \%$ da CVM, amostrados a $1024 \mathrm{~Hz}$ e submetidos a filtro passa-faixa com frequências de corte de 10 e $500 \mathrm{~Hz}$. No presente trabalho, foram utilizados sinais de EMG de contrações isométricas a $60 \%$ da CVM. Assim, para comparação, utilizou-se os resultados obtidos por Caroti et al. para 50\% e $70 \%$ da CVM. Os valores médios de MSE obtidos foram de 5,95\% (PRD $\cong 24,4 \%)$ e $5,26 \%$ (PRD $\cong 22,9 \%)$, respectivamente. O fator de compressão (FC) da configuração testada era de $87,3 \%$.

Dos modos de codificação testados no presente trabalho, o modo 2LP/DA/DF10 é o que mais se assemelha ao modo utilizado por Carotti et al. Esse modo tem o mesmo CF de $87,3 \%$ e propiciou um MSE médio de $5,5 \%$ (PRD $\cong 23,0 \%$ ) para os sinais de teste utilizados nesse trabalho. Ou seja, o modo 2LP/DA/DF10 tem desempenho equivalente ao do modo utilizado por Carotti et al.

Entretanto, com a retirada do dicionário adaptativo realizada no modo 2LP/DF10, o desempenho mostrou-se melhor que o obtido por Carotti: o MSE reduziu para 3,49\% e o CF aumentou para 89,7\%. Além disso, com o modo 1LP/DF10 se conseguiu um CF de 90,3\% e um MSE de 3,68\%. Por outro lado, com o acréscimo do segundo dicionário fixo, resultando no modo 2LP/DF10/DF10, se conseguiu reduzir o MSE para $1,70 \%$, embora, com a inclusão dos bits de codificação do dicionário fixo, o CF tenha caído para 81,35\%.

A comparação dos resultados acima mencionados está resumida na Figura 6.

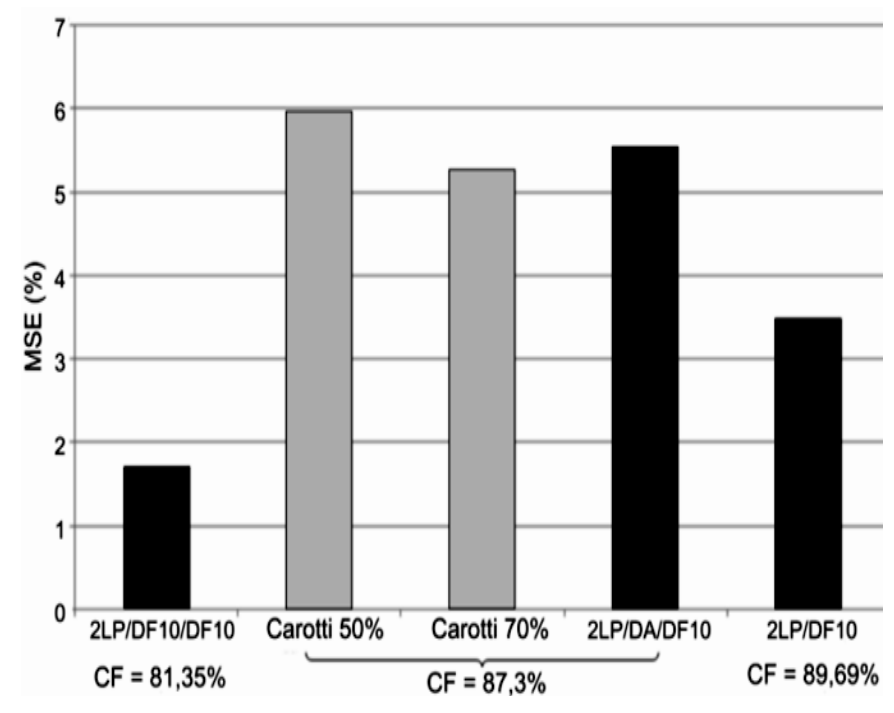

Fig. 6 - Comparação entre algunsdos resultados do presente trabalho e dos obtidos por Carotti et al. [7] para compressão de sinais de SEMG usando AMR-ACELP.

\section{H. Comparação com Outras Técnicas de Compressão}

Diferentes técnicas de compressão de EMG foram utilizados por Berger [6], que utiliza redes neurais e wavelets, e Costa et al [8], que utilizou o algoritmo de compressão de vídeo H.264/AVC. Como no presente trabalho, todos os sinais utilizados foram obtidos a partir de contrações isométricas. 
Uma comparação entre os valores obtidos com cada técnica pode ser vista na Figura 7.

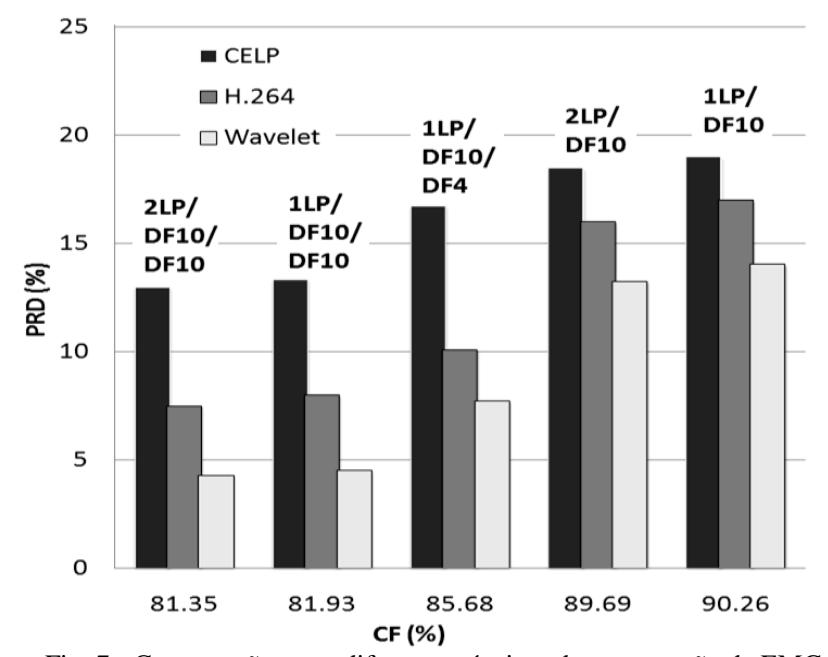

Fig. 7 - Comparação entre diferentes técnicas de compressão de EMG.

Em geral, os erros obtidos neste trabalho (CELP) são maiores que o obtido por outras técnicas, sendo esta diferença menor para a técnica utilizando H.264/AVC. Em particular, para as duas maiores taxas de compressão, correspondente à região em que as técnicas de Berger e Costa começam a produzir um aumento maior dos erros, as diferenças são menores.

\section{CONCLUSÕES}

Procurou-se avaliar neste trabalho a viabilidade da utilização de codificadores baseados no modelo CELP, na codificação de sinais de eletromiografia. Os resultados obtidos foram comparáveis e em alguns casos melhores que os obtidos por outras pesquisas utilizando a mesma ou outras técnicas de compressão.

Houve um aumento na qualidade objetivamente medida dos sinais reconstruídos quando se retirou os fatores que reforçam a qualidade de voz subjetivamente percebida e o dicionário adaptativo, e com a inclusão de um segundo dicionário fixo. Foi avaliada a variação de qualidade em função do tipo e quantidade de dicionários e conjuntos de parâmetros lineares utilizados.

Deve ser ressaltada a relativamente baixa complexidade e eficiência obtidos com o uso de código derivado do AMR original, especialmente devido ao algoritmo ACELP, comparativamente a outras técnicas de compressão de sinais. A simplicidade aumentou no código empregado neste trabalho após a exclusão do dicionário adaptativo. Sabe-se que o codec AMR é adequado para aplicações em tempo real e na implementação de dispositivos de comunicação móvel de terceira geração, tais como telefones celulares e outros dispositivos portáteis com chips DSP, sugerindo que pode ser adaptado com sucesso para o uso em dispositivos de processamento de EMG e talvez outros sinais eletrofisiológicos.

\section{AGRADECIMENTOS}

Agradecemos ao Professor Pedro Berger (CIC-UnB) por fornecer os sinais de EMG utilizados neste trabalho e a Letícia Neumann pelo apoio na elaboração dos gráficos.

\section{REFERÊNCIAS}

[1] R. Merletti and P. A. Parker, Electromyography: Physiology, Engineering and Noninvasive Applications, USA: Wiley-Interscience, 2004.

[2] 3rd Generation Partnership Project; Technical Specification Group Services and System Aspects; Mandatory speech CODEC speech processing functions; AMR speech CODEC; General description, 3GPP TS 26.071 V7.0.1 Technical Specification, 2007-07.

[3] J.-P.Adoul, P. Mabilleau, M. Delprat and S. Morissette, "Fast CELP coding based on algebraic codes", IEEE International Conference on Acoustics, Speech, and Signal Processing - ICASSP '87, Volume 12, Apr 1987 Page(s): 1957 - 1960.

[4] 3rd Generation Partnership Project; Technical Specification Group Services and System Aspects; Mandatory Speech Codec speech processing functions; Adaptive Multi-Rate (AMR) speech codec; Transcoding functions, 3GPP TS 26.090 V7.0.0 Technical Specification, 2007-06.

[5] 3rd Generation Partnership Project; Technical Specification Group Services and System Aspects; ANSI C code for the floating-point Adaptive Multi Rate (AMR) speech codec, 3GPP TS 26.104 V 7.0.0 Technical Specification, 2007-06.

[6] P. Berger, F. Nascimento, J. Carmo and A. Rocha, "Compression of EMG signals with wavelet transform and artificial neural networks", Physiol. Meas. 27 457-465, 2006.

[7] E. Carotti, J. Martin, R. Merletti and D. Farina, "Compression of surface EMG signals with algebraic code excited linear prediction", Medical Engineering \& Physics, Volume 29, Issue 2, Pages 253-258, March 2007.

[8] M. Costa et al, "Compressão de sinais de eletromiografia de superfície usando H.264/AVC", XI Congresso Brasileiro de Informática em Saúde, 2008.

[9] J. H. A. Cavalcante, "Compressão de Sinais Eletromiográficos usando Codificador Multitaxa Preditivo Adaptativo", Diss. de Mestrado, Universidade de Brasília, 2009.

[10] L. M. Silva, "Contribuições para a Melhoria da Codificação CELP a baixas taxas de bits", Tese de Doutorado. Pontifícia Universidade Católica do Rio de Janeiro, 1996.

[11] A. M. Kondoz, Digital Speech: Coding for Low Bit Rate Communication Systems, Second Edition, England: John Wiley \& Sons, Ltd., 2004. 